\section{Eisenüberladung als Folge regel- mäßiger Bluttransfusionen bei MDS-Patienten}

Patienten mit myelodysplastischen Syndromen, einer Gruppe erworbener klonaler Stammzellerkrankungen, sind häufig im Verlauf ihrer Erkrankung auf regelmäßige Bluttransfusionen angewiesen. Diese sind lebensnotwendig, können jedoch bereits nach kurzer Zeit - bei einer Frequenz von 2 Transfusionen pro Monat nach etwa 10 Monaten - zu einer Eisenüberladung führen. Um diese frühzeitig zu erkennen, sprechen sich Experten für ein regelmäßiges Monitoring des Serum-Ferritinspiegels aus. Ein Consensus Statement der MDS Foundation's Working Group on Transfusional Iron Overload empfiehlt eine Eisenchelat-Therapie ab einem Serum-Ferritinspiegel von $1000 \mathrm{ng} / \mathrm{ml}$ [1].

Zur Behandlung einer Eisenüberladung stehen die 3 Eisenchelatoren Deferasirox, Deferipron und Desferal zur Verfügung. Deferasirox $\left(\right.$ Exjade $\left.^{\circledR}\right)$, der erste einmal täglich oral einzunehmende Eisenchelator, ist seit ca. 2 Jahren in Deutschland auf dem Markt und im Allgemeinen gut verträglich. Deferasirox bietet einen 24-Stunden-Schutz gegenüber der gewebszerstörenden Wirkung des durch Eisenüberladung verursachten oxidativen Stresses und reduziert die Eisenlast sowohl in der Leber als auch im Herz.

Aktuelle Studie zu Deferasirox bei polytransfundierten MDS-Patienten

Derzeit rekrutiert die offene, einarmige Phase-III-Studie CICL670ADE03 erwach-

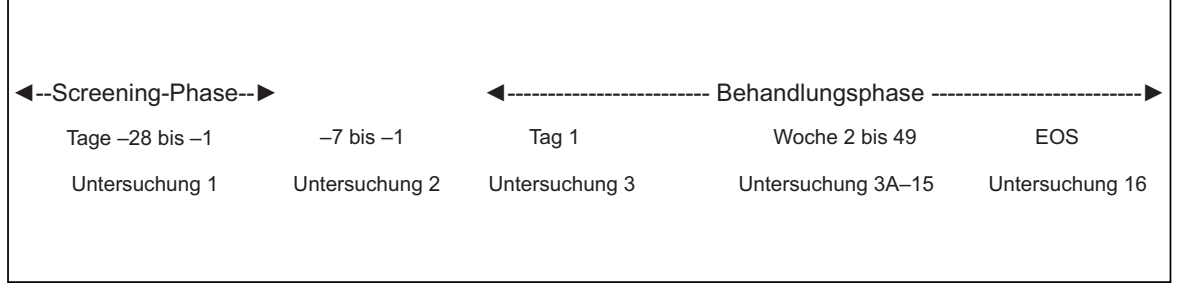

Abb. 1. Studiendesign CICL670ADE03.

sene MDS-Patienten der IPSS-Risikogruppen «niedrig» oder «Intermediär-1». Im Fokus dieser multizentrischen Studie steht die Untersuchung der Wirksamkeit, Verträglichkeit und Sicherheit von Deferasirox in der Behandlung von Patienten mit MDS und transfusionsbedingter Eisenintoxikation. Ziel ist es, insgesamt 120 erwachsene Patienten in die Studie einzuschließen. Voraussetzung - neben IPSS-Status und Volljährigkeit - ist ein Serum-Ferritinwert von mindestens $1000 \mathrm{ng} / \mathrm{ml}$ nach mindestens 20 Erythrozytenkonzentrat-Transfusionen. Wirksamkeits- und Verträglichkeitsparameter werden während der einjährigen Behandlungsdauer zu insgesamt 16 Untersuchungszeitpunkten erfasst (Abb. 1).

Primäres Ziel ist die Untersuchung einer wirksamen Eisenchelation, erfasst und bestätigt durch die Messung des SerumFerritinspiegels. Als sekundärer Zielparameter wurde unter anderem das Verträglichkeitsprofil, inklusive Dokumentation der Häufigkeit und Intensität von Diarrhöen, definiert. Untersuchungen zum Sicherheitsprofil erfassen neben Leber- und Nierenfunktionstests auch die Eisenkonzentration der Leber. Zusätzlich untersucht die CICL670ADE03-Studie, ob der Transfusionsbedarf unter einer Eisenchelat-Therapie mit Deferasirox sinkt [2].

\section{Referenzen}

1 Bennett JM, for the MDS Foundation's Working Group on Transfusional Iron Overload: Consensus statement on iron overload in myelodysplastic syndromes. Am J Hematol 2008; DOI: 10.1002/ajh.21269.

2 Novartis: A one-year, open-label, single-arm, multi-center trial evaluating the efficacy and safety of oral ICL670 in patients diagnosed with low and INT-1 risk myelodysplastic syndrome (MDS) and transfusion-dependent iron overload. Working protocol incorp., amendment 2. Novartis, November 22,2007

Weitere Informationen bei

Novartis Pharma GmbH

Dr. Oliver Leismann

Roonstraße 25, 90429 Nürnberg

Tel.+49 911 27312-247, Fax -739

oliver.leismann@novartis.com

\title{
Ticker+++ Ticker+++ Ticker+++ Ticker+++ Ticker+++ Ticker+++ Ticker+++
}

Alexion hat für Soliris ${ }^{\circledR}$ (Eculizumab) den «Prix Galien USA 2008» als bestes Biotechnologie-Produkt erhalten. Der Preis würdigt die wissenschaftliche Innovation der Komplement-Inhibition von Soliris ${ }^{\circledR}$ und deren Bedeutung für Patienten mit Paroxysmaler Nächtlicher Hämoglobinurie (PNH), einer seltenen, schweren und lebensbedrohlichen Bluterkrankung.

Weitere Informationen bei

Alexion Pharma Germany GmbH

Melanie Hansen

Landsberger Straße 155, 80687 München

Tel. +49 89 57959-674, Fax -214
Octapharma hat die Zulassung für octagam ${ }^{\circledR}$ $10 \%$ erhalten. Für das neue polyvalente Immunglobulin zur intravenösen Infusion ist es durch den Einsatz innovativer Technologien gelungen, auf der Basis von octagam ${ }^{\circledR}$ die Konzentration auf $10 \%$ zu erhöhen, ohne die bewährte Galenik zu verändern, auf der die hervorragende Verträglichkeit von octagam ${ }^{\circledR}$ basiert.

Weitere Informationen bei

Octapharma GmbH

Elisabeth-Selbert-Str 11, 40764 Langenfeld

Tel. +49 2173 917-0, Fax -11
Bayer. Die Europäische Kommission hat die Marktzulassung für Xarelto ${ }^{\circledR}$ (Rivaroxaban) erteilt. Der neuartige Gerinnungshemmer wird einmal täglich als Tablette eingenommen und kann nun zur Prophylaxe von venösen Thromboembolien (VET) bei erwachsenen Patienten nach elektiven Hüft- oder Kniegelenkersatzoperationen eingesetzt werden. Die Vermarktung in den EU-Mitgliedsstaaten beginnt in Kürze.

Weitere Informationen be

Bayer HealthCare AG

Dr. Jost Reinhard

Müllerstraße 178, 13353 Berlin

Tel. +49 30 468-15062 
INTERCEPT BLOOD SYSTEM ${ }^{T M}$ - Pathogen-

inaktivierung in Europa

\section{Neue Strategie für mehr Sicherheit bei Bluttransfusionen}

Trotz vieler Bemühungen, die pathogene Belastung von Spenderblut zu reduzieren oder betroffene Einheiten zu identifizieren, führen kontaminierte Blutprodukte nach wie vor $\mathrm{zu}$ transfusionsbedingten Nebenwirkungen bis hin zu schweren Erkrankungen. In einem Satellitensymposium im Rahmen der gemeinsamen Jahrestagung der DGTI und der französischen Gesellschaften SFTS und ESFH Mitte September in Düsseldorf, gesponsert von CERUS Corporation, war dies Gegenstand mehrerer wissenschaftlicher Vorträge. Exemplarisch für die Situation weltweit, so Dr. med. B. Höchsmann vom DRK Ulm, dokumentieren Daten, die von 1994 bis 2006 in Frankreich im Rahmen des nationalen Hämovigilanz-Systems erhoben wurden, den genannten Sachverhalt. Ein signifikantes Restrisiko der Kontamination von Blutprodukten mit pathogenen Keimen trotz konventioneller Sicherungssysteme mache eine Sensibilisierung für diese Problematik ebenso notwendig wie die Überlegung, ein aktives Verfahren der Pathogeninaktivierung in den Herstellungsprozess von Blutprodukten einzubinden.

Dr. Höchsmann verwies auf aktuelle europäische und nordamerikanische Studien, die untersuchten, inwieweit konventionelle Techniken zum Nachweis bakterieller Kontamination ausreichend Schutz vor kontaminierten Thrombozytenkonzentraten boten. Sie kamen übereinstimmend zu dem Ergebnis, so Dr. Höchsmann, dass bei geringer Kontamination pathogene Keime erst nach etwa vier Tagen nachweisbar sind. Dann jedoch sei ein
Großteil der Konzentrate bereits transfundiert. Zudem träten gravierendere Nebenwirkungen auf, wenn man ältere Einheiten verabreichte. Dies korreliere - zusätzlich zu verringerter Qualität der Thrombozyten nach längerer Lagerung - mit der signifikant gestiegenen Keimbelastung, die bei erneuter Untersuchung von Einheiten nachgewiesen wurde, die zum früheren Zeitpunkt als nicht kontaminiert getestet worden waren.

Ein höheres Maß an Sicherheit zur Prävention der Transfusion kontaminierter Blutprodukte bietet das Intercept Blood System ${ }^{\mathrm{TM}}$, das von CERUS Corporation entwickelt wurde. Die Wirksamkeit des Verfahrens gegen Bakterien, Viren und Protozoen sowie zur Inaktivierung von Leukozyten wurde in präklinischen und klinischen Studien bewiesen. Das photochemische Verfahren verfügt seit mehreren Jahren für Thrombozyten- und Plasma-Konzentrate über die europäische CE-Zertifizierung sowie über erste Vermarktungsgenehmigungen in Deutschland und Frankreich und ist in vielen Instituten im Routineeinsatz. In Bezug auf die Pathogeninaktivierung von Erythrozyten-Konzentraten befindet es sich in der Phase klinischer Testung.

Fundierte Forschung weist nach, so erläuterte Dr. med. G. Andreu vom INTS in Paris, dass dieses Verfahren nicht nur die pathogene Kontamination von Blutprodukten und daraus resultierende unerwünschte Transfusionsreaktionen signifikant reduziert, sondern kosteneffizient konventionelle Verfahren zum Nachweis von Bakterien und Viren ebenso ersetzen kann wie Gamma-Bestrahlungen.

Die Ergebnisse einer Langzeiterprobung des Intercept Blood Systems ${ }^{\mathrm{TM}}$, so Dr. med. D. Kientz vom EFS in Straßburg, bestätigen diese Aussagen. Unter Berücksichtigung zahlreicher Tests der Intercept-behandelten Blutkomponenten bezüglich Zusammensetzung und therapeutischer Effizienz schlussfolgerte er, dass das Verfahren sich sehr gut an die Erfordernisse regionaler Blutbanken anpasst. Die in Strassburg erhobenen Daten belegen, so Dr. Kientz, eindrucksvoll das Mehr an Sicherheit durch die Gabe Interceptbehandelter Thrombozyten-Konzentrate anhand der gesunkenen Anzahl an unerwünschten Transfusionsreaktionen.

Für das DRK Baden-Württemberg/Hessen erläuterte Dr. med. R. Henschler die Einbindung des Intercept Blood Systems ${ }^{\mathrm{TM}}$ in den Alltag des größten deutschen Blutspendedienstes. Mit dem Verfahren erhalte man bakteriologisch/virologisch unbedenkliche, qualitativ einwandfreie, uneingeschränkt wirksame Plasma- und ThrombozytenKonzentrate schon in ca. 6 bis 8 Stunden. Daraus ergebe sich eine erweiterte Nutzungsdauer, was wiederum ökonomisch von Belang sei.

Lucia Hajduk, Berlin

\section{Quelle}

«The Blood Safety Paradigm has Shifted: Experiences with INTERCEPT Pathogen Inactivation in France \& Germany.» Symposium anlässlich der 41. Jahrestagung der Deutschen Gesellschaft für Transfusionsmedizin und Immunhämatologie 2008. Cerus Europe B.V., Düsseldorf, 18.09.2008.

Weitere Informationen bei

Cerus Europe B.V.

Country Manager Germany

Monika Bauhaus

Tel.+49 $451202-8887$

mbauhaus@cerus.com

www.cerus.com

www.INTERCEPTBloodSystem.com

\section{Ticker+++ Ticker+++ Ticker+++ Ticker+++ Ticker+++ Ticker+++ Ticker+++}

Celgene. Das biopharmazeutische Unternehmen hat für sein Produkt Revlimid ${ }^{\circledR}$ (Lenalidomid) den renommierten US-amerikanischen «Prix Galien 2008" in der Kategorie außerordentliche therapeutische Entwicklung erhalten. Revlimid ${ }^{\circledR}$ ist der wichtigste Vertreter einer neuen Wirkstoffklasse, den sogenannten Immunmodulatorischen Substanzen $\left(\mathrm{IMiDs}^{\circledR}\right)$, und wird bei hämatologisch-onkologischen Erkrankungen eingesetzt.

Weitere Informationen bei

Celgene $\mathrm{GmbH}$

Joseph-Wild-Straße 20, 81829 München

Tel. +4989451519-010
Ortho Biotech. Die europäische Arzneimittelzulassungsbehörde EMEA hat Ende August die Zulassung für Velcade ${ }^{\circledR}$ (Bortezomib) in Kombination mit Melphalan und Prednison (VMP) zur Primärtherapie von Patienten mit multiplem Myelom, die nicht für eine Hochdosischemotherapie mit autologer Stammzelltransplantation geeignet sind, erteilt.

Weitere Informationen bei

Ortho Biotech

Division of Janssen-Cilag $\mathrm{GmbH}$

Anne Wenders

Raiffeisenstr. 8, 41470 Neuss

Tel. +49 2137 955-0
Siemens Healthcare hat kürzlich für sein Immunoassay-System für mittelgroße klinische Labors, ADVIA Centaur CP, die Zulassung (CE) erhalten, in Europa mehrere Assays zur Bestimmung von Markern für Hepatitis-B-Viren zu vertreiben. Es handelt sich dabei um Tests auf Hepatitis-B-Oberflächen-Antigene und den HBsAg-Bestätigungstest. Außerdem zählen Antikörper gegen den Kern des Hepatitis-B-Virus dazu und die IgM-Antikörper gegen das Hepatitis-B-core-Antigen.

Weitere Informationen bei Siemens AG

Healthcare Sector - Diagnostics Division Henkestraße 127, 91052 Erlangen 